\title{
Intelligent Information Technologies to Enable Next Generation PLM
}

\author{
Rainer Stark ${ }^{1,2}$, Thomas Damerau ${ }^{1}$, Haygazun Hayka ${ }^{1}$, Sebastian Neumeyer $^{1}$, \\ and Robert Woll ${ }^{1}$ \\ ${ }^{1}$ Division Virtual Product Creation, Fraunhofer IPK, Germany \\ ${ }^{2}$ Chair of Industrial Information Technology, Technische Universität Berlin, Germany \\ thomas.dameraulipk. fraunhofer.de
}

\begin{abstract}
The steadily growing complexity of products, interfacing processes, value creation networks and IT environments drive today's PLM solutions to their limits. How does this effect engineers? Over 1,400 engineers from the German industry provided feedback in 2011 - with alarming but expected results. Almost two thirds of the respondents can only spend $20 \%$ or less time on average for core tasks such as development, design or validation. The study confirmed a lack of time for creative engineering activities caused by a massive coordination and communication overhead. Could engineers and designers be relieved from routine and administrative tasks in product lifecycle management by means of current "intelligent" technologies? In constant dialogue with industry and PLM experts, the Fraunhofer IPK and TUB have investigated the demand for intelligence in product lifecycle management. This paper reflects on the current situation of PLM and introduces a conceptual framework (Engineering Operating System) for next generation PLM. Subsequently, an Engineering Automation Capabilities (EAC) stair step model is proposed and selected research results for "intelligence" in PLM are presented.
\end{abstract}

Keywords: PLM, Next Generation, Engineering Operating System, Intelligence.

\section{$1 \quad$ Need for intelligent PLM Solutions}

Product Lifecycle Management (PLM) is an integrated approach to manage productrelated information along a product's entire lifecycle relying on a suitable set of organizational prerequisites, processes and IT solutions [1]. But with an ever growing complexity of products, processes, value creation networks and IT environments, managing all that information becomes an increasing challenge. Figure 1 depicts some of these challenges and drivers, identified by the authors based on experience in industrial and research projects.

To cope with these challenges from the IT perspective, PLM thus has to ensure that massive amounts of information can be stored, searched and used efficiently. Furthermore, PLM must ensure that information from different domains and lifecycle phases is logically and dynamically linked according to variable business intelligences. 

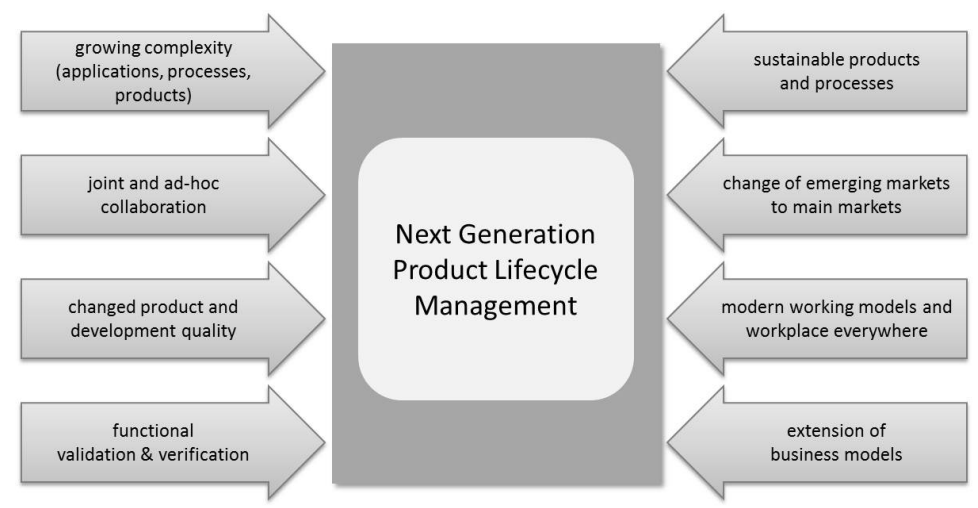

Fig. 1. Challenges and drivers for future PLM

However, current PLM solutions have three essential shortcomings addressed in this paper. Firstly, the underlying data models (describing how information from different engineering disciplines can be linked together) and the actual linking of information are left to the companies implementing a PLM solution into their IT environment. Secondly, PDM/PLM vendors have still not found or proposed a simple way for integrating third-party task-specific solutions besides the ones they offer themselves. And thirdly, almost any kind of "intelligence" in data management today is expected to be provided by the users (engineers, planners, managers). The first stated shortcoming of current PLM solutions calls for standardized, industry-wide accepted high-level PLM data models and for specific solutions that support the modelling of links between data from different domains and the analysis of interlinked data. The second shortcoming may not be obvious at first sight. It must be understood that task-specific solutions and the PDM backbone that they build upon are two types of IT solutions; such implementation oriented separation is necessary to allow for companies to choose the best breed of task-specific solutions and also allow them to develop their individual solutions if needed. In the following, these standalone task-specific solutions will be called PLM solutions, as opposed to PDM/PLM solutions which represent a tightly coupled, combined set of PLM solutions and a PDM backbone that are all offered by the same IT vendor. A future PLM environment has to allow for any PLM solutions to simply be plugged into an already existing PDM backbone. The third shortcoming of today's PLM solutions is characterized by the fact that no sort of intelligence is provided that could relieve engineers from some of the rather dull data-management-centric activities. This leads to an innovation backlog that was confirmed by a survey that 1,400 engineers from the German industry provided feedback to in 2012/2013 [2, 3]. Almost two thirds of the respondents could only spend $20 \%$ or less time on average for core tasks such as development, design or validation. The study confirmed a lack of time for creative engineering activities caused by a massive coordination and communication overhead. Due to these shortcomings this paper aims to introduce and review concepts and technologies that will lead to a more intelligent support of engineering activities and an automation of data management related activities in future PLM solutions. 
Research Approach. The development of intelligence in PLM solutions demands extensive research activities; a holistic consideration of industrial needs, methodical requirements, technical feasibility and other aspects is necessary for this. To cope with this challenge a research approach was defined in order to answer the research question: How should information technologies be used to best relieve engineers from routine tasks in PLM? The approach contains the following steps:

- proposal and validation of a holistic conceptual PLM framework,

- proposal of a stair step model for "intelligent" PLM solutions, and

- review of existing "intelligent" PLM building blocks and classification according to the stair step model for gap identification.

\section{Conceptual Framework for PLM Solutions}

This chapter introduces two models; first a conceptual framework for PLM, designated as Engineering Operating System (EOS) [5], proposed by Fraunhofer IPK in 2013, and second, a maturity stair step model, designated as Engineering Automation Capabilities (EAC) model, for PLM solutions. The EOS was drafted with the intention to ensure product creation capabilities in the context of a holistic framework. It is based on four different levels that need to be taken into consideration when PLM processes, solutions or strategies are conceived. The core message of the EOS is that these four different levels must be understood as an integrated system in order to unfold the complete potential of PLM. These four levels are characterized as follows (compare Fig. 2):

- Process and Organization: At this level it has to be ensured that roles, responsibilities, and processes exist that define which value creation activities are to be executed by whom and hence need to be supported by PLM solutions. Furthermore, the information needs of business processes and management must be understood in order to support an effective and efficient information ex-change between engineering activities and management activities.

- Engineering Activities: Engineering Activities generally comprise all activities during the lifecycle that contribute to the generation, verification and validation of a virtual model of a product and to accompany a physical product from its production down to its end of life. While the process and organization layer focusses on the high-level processes, dependencies and responsibilities and thus on the questions of when things are done, in which order and by whom, the engineering activities focus on "how" exactly things are done in detail. From a PLM perspective it must be ensured that engineers have the required access to product-related information when they perform engineering or service activities. In addition it must be ensured that engineers can define and store new information in a way that allows a later reuse of that information (where needed in the lifecycle). 
- Data and Information Models: On this layer PLM must thrive to understand the way in which different engineering stakeholders exchange information and data, how they interact with it from their different perspectives and how data is converted and filtered. PLM must ensure that information from different do-mains can be interlinked, that limited views on information can be established according to different application contexts and access policies, and that the lifecycle of data is managed.

- IT-Implementation: In order for all product-related information to be properly managed PLM must ensure that a suitable technological backbone for data management is in place (e.g. servers, networks, service platforms). This comprises the availability of reliable and safe data storages, the use of flexible, efficient and ideally open and standardized data formats, the provision of powerful and user-friendly information retrieval interfaces and the realization of efficient IT system integration mechanisms.

Yet accomplished innovations in PLM solutions were mainly driven from an operational perspective and therefore mostly focused on the top layer "Process and Organization". Examples are the inclusion of project-, workflow management or applications for the conversion of digital models. The engineering activity level is almost not addressed at all. PLM solutions barely support engineers in their key activities such as technical assessment, alternative solution identification and creation, technical system artefact traceability, decision making but mainly address administrative tasks and digital model management.

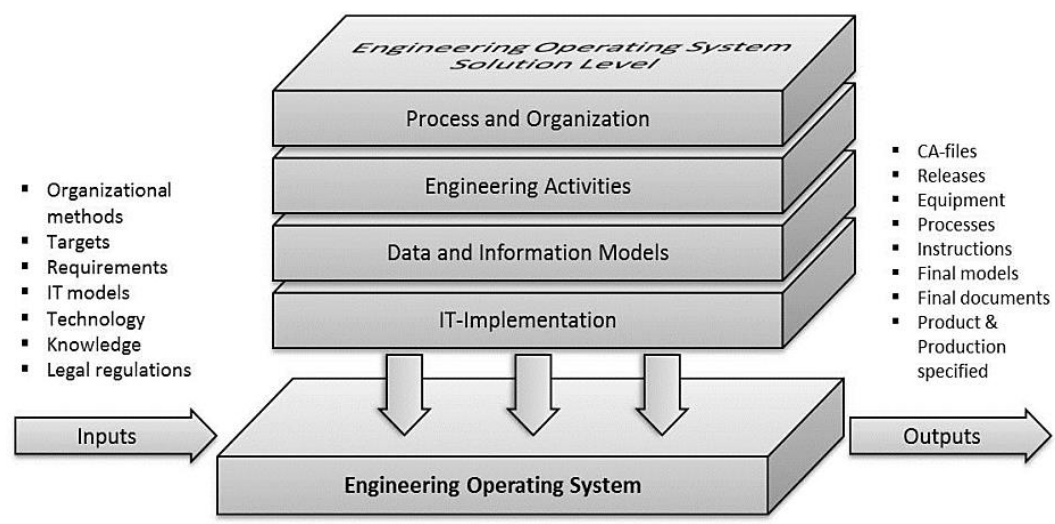

Fig. 2. Solution Layers of the conceptual Engineering Operation System (EOS) model

At the data and information models layer, many PLM solutions, however, still employ proprietary data formats that hinder a fluent information exchange across organizations, and at the "IT-Implementation" layer they usually do not provide open 
programming interfaces that would allow them to be easily coupled with other PLM solutions. The authors suggest that the use of intelligent information technologies may improve the degree of integration across PLM solutions, have a positive impact on the holistic integration of the two top layers and lead to both, an increased engineering effectiveness and efficiency, and a relief of engineers form routine tasks.

To gain clarity and for the classification of existing and targeted next generation PLM solutions, a stair step maturity model is proposed. The Engineering Automation Capabilities (EAC) model describes the expected and required increments of engineering automation capabilities towards intelligent PLM solutions in four steps (see Fig. 3.)

- User-centered Automation: This step represents today's state of the art in industry. PLM solutions consist of stand-alone PLM applications. Although PLM vendors offer partly integrated solutions e.g. integrated requirements management with linking functionalities to the product structure, these are not widely diffused into industrial practice. Hence, extensive customizing and configuration efforts are made. The engineer is the central source for the intelligent automation of data management centric activities in PLM.

- Assisted Automation: This second level offers developments towards intelligent approaches for data and information management. The engineer is still the single source of intelligence, but is assisted by tools during data search, link-age, usage and other data management centric activities.

- Semi-automated Engineering: Semi-automated engineering means that PLM solutions are able to anticipate engineering activities (e.g. action alternatives and data aggregation for the configuration of a digital mockup). This is the first step towards artificial intelligence (AI) in PLM. Semi automation already uses inherent business intelligences to trigger new engineering tasks or at least information related analysis, synthesis or assessments.

- Automated Engineering: The last step is enabling the automation of routine engineering activities and data management centric activities. Data and information is linked both, vertical through all solution levels of the EOS and the involved domains (mechanic, electronic and informatics), and horizontal along the product lifecycle. Therefore, the anticipation of engineering activities can be enhanced. Furthermore consequences of decisions can be estimated and aligned with business and engineering objectives automatically.

The step "automated engineering" in the stair step model can be seen as the ultimate goal. The next section elaborates present existing research results in context of the EOS and the associated stair step model. 


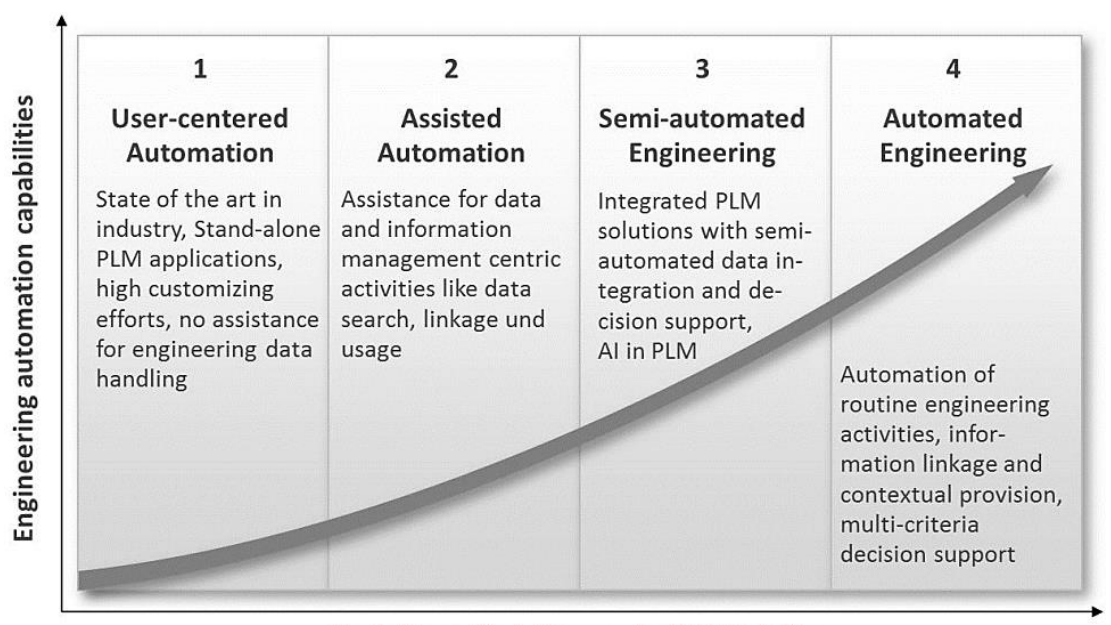

Evolution of intelligence in PLM Solutions

Fig. 3. Evolution steps for intelligent PLM solutions in the context of engineering automation capabilities

\section{Research Solutions for Intelligence in PLM}

In this section intelligent PLM-solutions are presented that have been developed at Fraunhofer IPK and the Chair of Industrial Information Technology at the Berlin University of Technology.

As described in the previous chapter, past PLM initiatives focused on the layer of "Process and Organization" of EOS. Also the layer of "Data and Information Models" is covered by PLM Solutions, even if the integration of heterogeneous data into PLM still has to be performed by an engineer or by customization and configuration effort in PLM-Solutions. Due to new methods like Systems Engineering (SE), but also due to the increased amount of data and information in the product lifecycle management processes, intelligent PLM-solutions also have to address intelligent solutions for the EOS layer of "Engineering Activities" as well as increased assistance and automation in "Data and Information Modeling" layer. Such solutions and their application in PLM are now presented and allocated in the introduced EAC stair step model.

Visual Analytics for PLM. According to Thomas \& Cook, visual analytics is an approach to understand past and current situations, as well as the events that lead to these circumstances. Likewise, visual analytics support the visualization of multiple data layers, the abstraction and integration of data from multiple data sources into a common representation [5]. This especially suits the needs in heterogeneous PLM IT environments, as today's PDM-Systems, which are used as backbone for PLM, including heterogeneous data as well as multiple levels of data layers. But such an amount of data and information can barely be controlled by the engineer. 
In Figure 4, an approach developed at the Chair Industrial Information Technology at the Berlin University of Technology is shown, where a potential delay in the development process of a product is analyzed with the support of visual analytics. To analyze or assess such a risk, the data stored in the PDM-System, e. g. the number of design iterations (revisions or engineering changes) done in a past project, is analyzed. By dynamic filtering of the number of iterations (threshold) of an object (see Fig. 4 a, b, c), the engineer receives direct visual feedback of similar challenges within the current project (P1) as well as the past project (P2). By applying a second indicator on the selected data in the PDM System, delay in design, it is possible to identify all those objects with the same level of criticality in the design process (see Figure $4 \mathrm{~d}$ ). The relevant object, a single part or an assembly, can be further investigated with the retrieved detail information (Figure 4e) and referenced or highlighted for the next risk review.

Other applications of visual analytics are e.g. to understand the chronology of change requests using a 3D product model or the visualization of impacts of changed parameters of a system model on a linked geometrical model. Visual Analytics provides intelligent support to information retrieval and thus fits into the 2nd step "Assisted Automation" of the EAC.

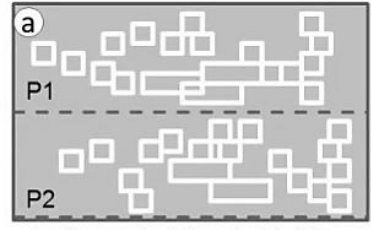

Indicator 1 - Threshold: 10

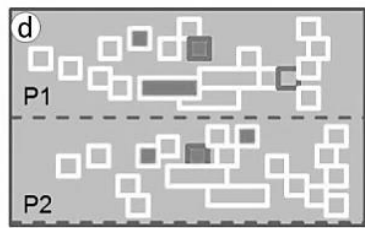

Indicator 2-complex geom.

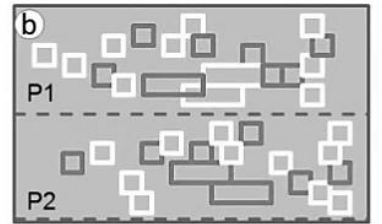

Indicator 1 - Threshold: 4

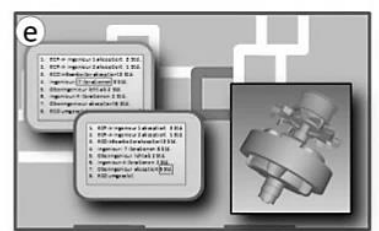

Retrieving detailed Information

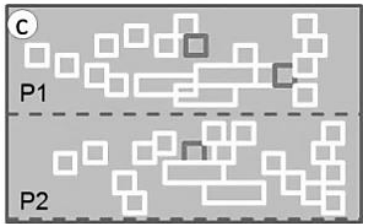

Indicator 1 - Threshold 6

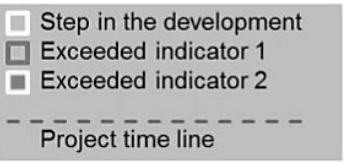

Project time line

Fig. 4. Example for an indicator based comparison of two projects using Visual Analytics

Traceability in Systems Engineering. Today, the intelligent engineering work in Systems Engineering (SE) covers the task of comprehension of the different domain models as well as the identification of the relevant parameters, requirements and interdependencies between do-main models. To handle this complexity, today's SE approaches propose the creation of tracelinks.

In order to improve the efficiency of tracelink modeling, the "Model Tracer" application has been developed within the research project ISYPROM ${ }^{1}$. This modeler allows the creation and analysis of links between isolated data artifacts (Figure 5) to

${ }^{1}$ Research project ISYPROM. URL: http: / /www. isyprom. de/ 
ensure consistency and to identify dependencies between data artifacts as well as to estimate the impact of engineering changes. Also other application scenarios like the Fault Tree Analysis, Failure Mode and Effects analysis, Pareto Analysis and tracelink based progress monitoring can be supported using the "ModelTracer" [6].

With a consequent tracelink approach, information on the interdependencies between domain models and other artifacts is made explicit thus allowing for enhanced data analysis. The analysis of such interdependencies would otherwise have to be performed manually by the engineer. Given the amount of different models this is a tedious activity with a high risk of overlooking implicit interdependencies. The "Model Tracer" thus also improves the reliability and efficiency of analyzing model interdependencies. Hence, it mainly addresses the "Data and Information Models" layer of the EOS. Providing intelligent support at data level it also fits step 2 of the EAC.

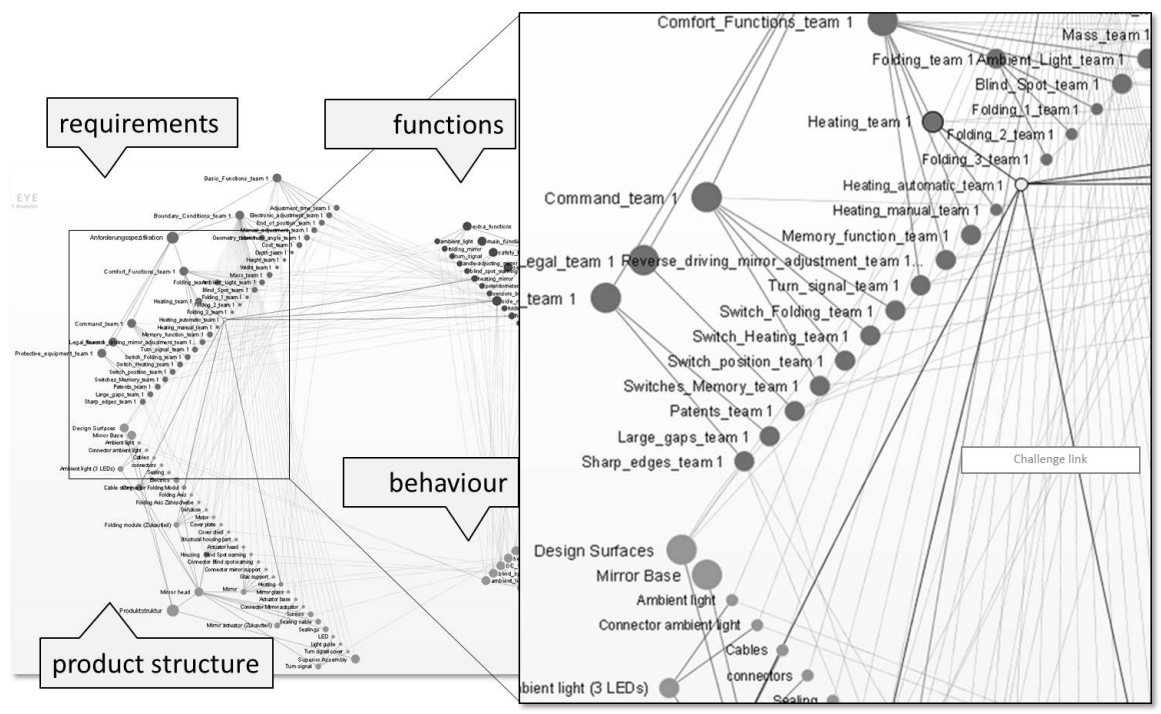

Fig. 5. Graphical presentation tracelinks that have been create with the "ModelTracer"

Ontologies for Semantic Data Integration. An important prerequisite before the ModelTracer can be used, is the definition of a data metamodel that defines which types of tracelinks can be defined between which elements from different data models. In the research field of data integration, a data metamodel that specifies possible relations between heterogeneous data formats is called a "mediated schema". In the EU-funded research project iProd ${ }^{2}$ Fraunhofer IPK has investigated the use of ontologies as mediated schemas. The goal of these schemas was not only to allow for tracelink modelling but also for providing a technological foundation that allows for intelligent algorithms (so-called "reasoners") to process existing engineering data. These reasoners shall discover logical links between heterogeneous product data automatically in order to reduce the time for manual tracelinking even further than the ModelTracer allows.

${ }^{2}$ Research project iProd URL: http: / /www. iprod-project. eu 
In the iProd project Fraunhofer IPK has developed ontologies specifying a highlevel engineering metamodel for interlinking different engineering artefacts that are generated and used along the Product Development Process (PDP). These ontologies reuse many concepts known from SysML and STEP 233 and they are designed to be easily adaptable for different engineering domains (such as the automotive, aerospace or home appliances domains) [7]. When adapted to the specific vocabulary of one company, an additional ontology-based data integration framework allows for specifying interfaces for different PLM solutions. Through these interfaces, existing engineering data can be linked and subsequently be processed by reasoning algorithms. Using reasoning, not only tracelink modelling can be sped up but also other logical rules can be applied to engineering data. For example, consistency rules between different behavior simulation models could be defined that allow for automatic model checking through a reasoner. Hence, by mapping non-ontological data to an ontology as a mediated schema, additional semantics can be "added" to existing engineering data without modifying it.

Decision Making Support for Sustainable Product Design. For dedicated tasks in engineering, e.g. for sustainable product design, the interdependencies between design decisions during development and sustainability effects of corresponding value creation are so complex, an engineer can't estimate them without support [8]. Currently, at the Chair of Industrial Information Technology at the Berlin University of Technology, an assistance system for semi-automated decision assistance in sustainable product design is under development in the research project CRC1026 ${ }^{3}$.

For this approach, several concepts for decision support have been investigated. One approach is to use ontologies for interlinking data from different phases in the product lifecycle. The sustainability information handled today in many different ITSystems and databases are combined with knowledge artefacts based on sustainability related methods (Figure 6). With the help of several data bases, different methods as well as aggregated, sustainability-related knowledge, an assessment of different design alternatives is possible.

Another approach is the usage of a knowledge base, where the core functionality of the support system is the classification of the various properties and characteristics of a product with respect to its sustainability. For this, the environmental and social impacts over the entire lifecycle are considered and information is gathered about the sustainability context areas, e. g. the manufacturing processes and equipment, and about re-use. The system evaluates both designer's intention and the stakeholder's needs and requirements by evaluation criteria. [9].

Such semi-automated solutions enable the engineer to gather all stored knowledge and make the right decisions by pre-defined rules. Nevertheless, even if the 2nd and 3rd step of EAC can be achieved through interlinking the layers "engineering activity" and "data and information models" of the EOS, additional re-search is necessary in order to achieve the 4th step in the EAC model, the "Auto-mated Engineering" as the final evolution in assistance capabilities.

\footnotetext{
${ }^{3}$ Collaborative Research Center 1026 Sustainable Manufacturing - Shaping Global Value creation URL: http: / /www.sustainable-manufacturing.net/en_GB/
} 


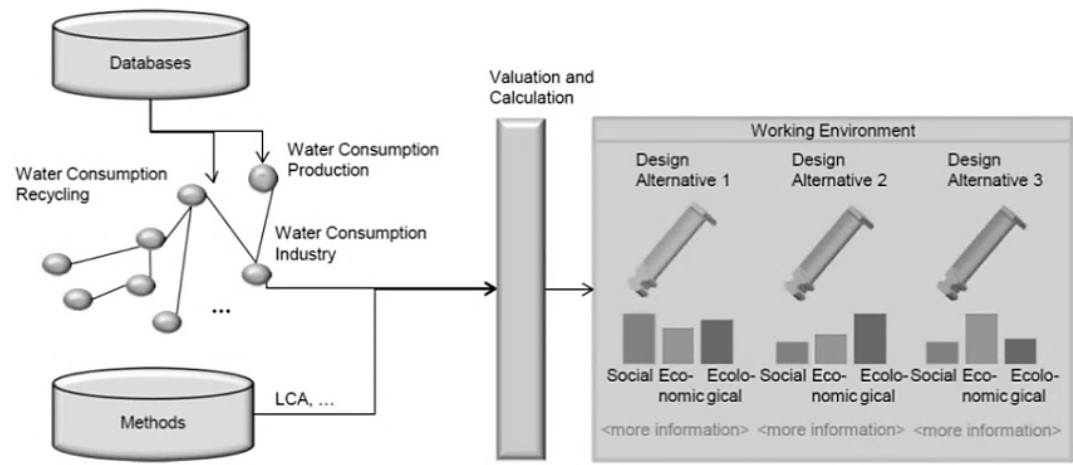

Fig. 6. Approach for integrating sustainability information into an engineer's working environment [6]

\section{Conceptual Framework for PLM Solutions}

In this paper it has been argued that engineers spend too much time on nonengineering-centric activities. The presented EOS suggests that PLM must always consider four different layers and ensure that these are harmonized. Furthermore, it postulates that PLM must assist engineering activities by providing the necessary information to engineers and that data management centric activities should be automated as much as possible. The EAC stair step model defines the different degrees to which PLM may employ intelligent approaches in order to reach these goals.

Fraunhofer IPK and TUB are introducing technologies and approaches for more intelligent PLM solutions. While a set of promising, existing research results are presented, a coherent vision for, and realization of, future PLM, that addresses all layers of the EOS, is still under development. The essence of that vision is to make better use of artificial intelligence approaches and to build a more integrated PLM IT environment. This future vision can only be reached if research, IT vendors, and industry end users work hand in hand. Towards this, further research steps will be taken by Fraunhofer IPK and TUB:

- Application of a scenario technique [10] to identify major challenges for intelligent data management from the industry perspective and definition of use-cases for the application of intelligent management.

- Implementation of a PLM based simulation environment to quantify and qualify the impact and synergies of presented and further approaches in the context of defined use-cases.

This paper is a contribution to the research field of next generation PLM, in awareness of the need for intensified interdisciplinary scientific discourse. Growing PLM user demands for more intelligence in PLM can be best satisfied by the collaborative research with PLM vendors and PLM users as well. 


\section{References}

1. Stark, J.: Product Lifecycle Management: 21 st Century Paradigm for Product Realisation, 572 p. Springer-Verlag London Limited, London (2011)

2. Müller, P., Pasch, F., Drewinski, R., Hayka, H.: Kollaborative Produktentwicklung und digitale Werkzeuge: Defizite heute - Potenziale morgen, 103 p. Fraunhofer Institut Produktionsanlagen und Konstruktionstechnik, Berlin (2013)

3. Müller, P., Pasch, F., Drewinski, R., Bedenbender, H., Hayka, H., Stark, R.: Study on collaborative product development and digital engineering tools. In: Rivest, L., Bouras, A., Louhichi, B. (eds.) PLM 2012. IFIP AICT, vol. 388, pp. 389-399. Springer, Heidelberg (2012)

4. Stark, R., Hayka, H., Rothenburg, U., Figge, A., Woll, R.: Keynote presentation: Next Generation Smart Product and Systems Engineering; COBEM. In: International Congress of Mechanical Engineering, University of São Paulo, Ribeirão Preto, Brazil (2013)

5. Thomas, J.J. (ed.): Illuminating the path: the research and development agenda for visual analytics, 186 p. IEEE, Los Alamitos (2005)

6. Beier, G., Figge, A., Müller, R., Rothenburg, U., Stark, R.: Supporting Product Development through Cross-Discipline Dependency-Modeling: Novel Approaches for Traceability-Usage. Lecture Notes on Information Theory 1(1), 21-28 (2013)

7. Woll, R., Hayka, H., Stark, R., Geißler, C., Greisinger, C.: Semantic integration of product data models for the verification of product requirements, in: Concurrent engineering approaches for sustainable product development in a multi-disciplinary environment. In: Proceedings of the 19th ISPE International Conference on Concurrent Engineering, pp. 157-168. Springer, London (2013)

8. Pförtner, A., Lindow, K., Hayka, H., Stark, R.: Concept for decision-making support in sustainable product design based on semantic ontology. In: Proceedings: 10th Global Conference on Sustainable Manufacturing Towards Implementing Sustainable Manufacturing, Istanbul, Turkey, October 31 - November 2, pp. 476-480. Springer, Heidelberg (2012); Towards implementing sustainable manufacturing. Proceedings

9. Lindow, K., Heimann, O., Adolphy, S., Hayka, H., Stark, R.: Decision-Making Support for Sustainable Product Development. In: Abramovici, M., Stark, R. (eds.) Proceedings of the 23rd CIRP Design Conference on Smart Product Engineering, Bochum, Germany, March 11-13, pp. 979-988. Springer, Berlin (2013)

10. Gausemeier, J., Plass, C., Wenzelmann, C.: Zukunftsorientierte Unternehmensgestaltung: Strategien, Geschäftsprozesse und IT-Systeme für die Produktion von morgen, 467p. Hanser, München, (2009) 